\title{
A Study on Negative Transfer of Non-English Majors' Writing Under the Error-Analysis Theory
}

\author{
Xiaohua $\mathrm{Mi}^{1, \mathrm{a}^{*}}$, Wanqing Chen ${ }^{2, \mathrm{~b}}$, Yan Zhang ${ }^{3, \mathrm{c}}$ \\ ${ }^{1}$ School of Foreign Languages, Xidian University,Xi'an, Shaanxi, China \\ ${ }^{2}$ School of Foreign Languages, Xidian University,Xi'an, Shaanxi, China \\ ${ }^{3}$ School of Foreign Languages, Xidian University,Xi'an, Shaanxi, China

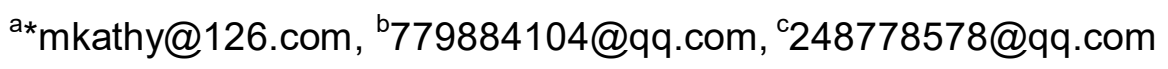

Keywords: Negative transfer; College English writing; Strategy; Avoid

\begin{abstract}
In second language acquisition, one's mother tongue will influence both in a positive and negative way. While Chinese and English belong to different language families and have different cultural background, negative transfer appears in vocabulary, sentence construction, coherence, and culture. In this paper, the author studies negative transfer in college English writing. 150 sophomores from Xidian University are chosen, and interview and writing test are combined to accomplish the study. After statistical analysis of the students' negative transfer in the writing test, the author explores the appearance of them in college English writing, and proposes feasible strategies to avoid negative transfer.
\end{abstract}

\section{Introduction}

In English writings of students whose mother tongue is Chinese, there are quite a lot of errors caused by the influence of mother language, which is called negative transfer of mother language.

In so many years' teaching experience, we find that errors committed by non-English majors in their writing are quite typical and can be classified. So we intend to use the error analysis theory to analyze the mistakes in college students' writing, and then based on the result of the analysis, we propose some strategies to help students reduce or even avoid those kind of errors.

\section{Theoretical background}

Negative Transfer Theory. Transfer was originally a psychological term which comes from the Latin word "transferre" meaning "bear, transfer, or copy". With the change of its meaning in practice and use, it comes to mean "to carry or generalize the acquired skills to other learning process, especially knowledge, skill, and proficiency". Later on, "transfer" was used in language acquisition. In language acquisition, transfer is defined as the use of acquired language in new process of learning, spontaneously, uncontrollably, or subconsciously. The influence of acquired language on new learning process can be divided into two categories: positive transfer and negative transfer. Positive transfer helps in the new learning process, while negative transfer, as its name suggests, hinders. That's why we should study it based on certain theories, and put up strategies to hale college non-English majors to reduce negative transfer. 150 sophomores from different majors in Xidian University are chosen in this study, and their writings are analyzed.

Error analysis theory. Error analysis theory was proposed by Britain applied linguist S. Pit Corder in 1970 s. He said errors are unavoidable parts in learning ${ }^{[1]}$. Researchers studied and analyzed various errors in foreign language acquisition. The steps of error analysis theory include: (1)choose material; (2) confirm errors; (3)classify errors; (4)explain reasons; (5)evaluate its instructive meaning in teaching. While classifying errors, Richards divided the errors of second language learners into inter-lingual errors, intra-lingual errors, and developing errors. Inter-lingual errors refer to errors caused by the influence of mother language, while the other two types are not related to mother language because they 
are caused by the learners' understanding and mastering of the target language ${ }^{[2]}$. Corder also thinks that errors in learners' language learning play a significant role in understanding and studying second language acquisition. To be specific, it helps in the following aspects: (1)to know learners' grasp of the language and what they should learn next ; (2)to know how language learners acquire languages and what strategies and steps they apply; (3)analysis of the errors are essential for the learners because learners can avoid similar errors, and thus to improve their learning ${ }^{[3]}$.

\section{Research Introduction}

Purpose of the research. By interviewing the students chosen and analyzing their writings, we will calculate their errors. By comparing the errors, we will examine the negative transfer of mother language on college English writing, and relative strategies are expected, which will be used in the teaching practice.

Research object. In this research, we randomly choose 150 students in Xidian University, and they come from 5 different schools: school of micro-electronics, school of mechanical and electrical engineering, school of communications, school of computer science, and school of physics and optoelectronics.

Research method. In this research, we combine interview and questionnaire (writing test). We interviewed a part of students from the 150 samples, and all the 150 students are invited to finish the questionnaires (we get 150 valid questionnaires). By collecting and analyzing the errors in their writings, we select those errors caused by negative transfer, and classify them for the research.

Research result. By randomly interviewing students and studying their writings, we classify and compare the errors caused by negative language, and then we get the following result.

Table 1 Result of the error analysis

\begin{tabular}{|l|l|l|}
\hline Error types & $\begin{array}{l}\text { Number } \\
\text { of errors }\end{array}$ & percentage \\
\hline $\begin{array}{l}\text { The meaning of } \\
\text { words }\end{array}$ & 152 & $11.1 \%$ \\
\hline Part of speech & 65 & $4.7 \%$ \\
\hline collocation & 236 & $17.2 \%$ \\
\hline Run-on sentence & 311 & $22.7 \%$ \\
\hline $\begin{array}{l}\text { Absence of tense } \\
\text { shifting }\end{array}$ & 228 & $16.7 \%$ \\
\hline $\begin{array}{l}\text { Ambiguity of topic } \\
\text { sentence }\end{array}$ & 133 & $9.7 \%$ \\
\hline $\begin{array}{l}\text { No-logical } \\
\text { connection among } \\
\text { paragraphs }\end{array}$ & 157 & $11.5 \%$ \\
\hline Cultural differences & 87 & $6.4 \%$ \\
\hline
\end{tabular}

Negative transfer of meaning of words. Negative transfer of vocabulary exits in different aspects: meaning, part of speech, collocation, and so on. In learning vocabulary, non-English majors often rely a lot on the help of their mother tongue, so they usually remember just the Chinese meaning of the words, and are not quite clear of their exact meaning in English. In this case, when using the vocabulary, they are not really aware of the words. When they want to use vocabularies, a Chinese word may come to their mind first, and then they translate the Chinese word into English before they use it. In fact, some words, white sharing similar meanings in Chinese, they are greatly different in English. For example, the student writes: My father is a common worker. The word "common" in this sentence, shares the seemingly same Chinese meaning with "ordinary". But actually, "common means "belonging to or shared by everyone in society" (Longman Dictionary of Contempory English), while "common" means 
"average, usual, not different or special". Obviously, "ordinary" is the correct word the student needs: My father is an ordinary worker.

Misuse of part of speech is not uncommon negative transfer. For example, the student writes: I againsted this ides. "Against" is a preposition, and can not be used as a verb. "I am against this idea" is the correct expression. Another example from the student: Then he closed to me and encouraged me. "close" means "shut" when it is used as a verb, but negative transfer from Chinese influences student to use it to mean "get near". What he wants to express is : He got close to me and encouraged me.

Besides, in collocation, negative transfer is also very common. Examples are as following: do your choice, open the light, repeat again. The correct expression should be: make your choice, turn on the light, repeat (say again). The negative transfer come from Chinese :做选择, 开灯, 再重复一次. This has been proved in the interview with the students.

Syntax negative transfer. English and Chinese belong to different language families. Chinese belongs to Sino-Tibetan language, so it emphasizes meaning itself but not so much on the form. While English belongs to Indo-European language family, and it emphasizes the form of language expression. Chinese students, when writing in English, from thinking to constructing a sentence, the influence of mother language is too obvious. In some of the incorrect sentences written by Chinese students, we can see clearly the sentence structure in a Chinese way. Sentences without proper tense changes are among the most common ones because in Chinese, tense is not reflected by verbs, but by some auxiliary words. For example: then he do what he said. In this sentence, the error was caused not just by the student's grammar, but the negative transfer from mother language.

Chinese is loose in structure, and the connection between two sentences are mainly reflected by the meaning itself. But in English, if two separated sentences are not connected by logical conjunctions, a run-on mistake will occur, as well as incorrect logical subject of the sentences. For example:

It was my mother stopped me. (there is no subject for the verb "stopped")

I was very nervous after heard this news. ("heard" should be "hearing")

Time goes by, but the thing happened in my childhood is still in my mind and will never forget. (the logical subject for " never forget" is "I", not "the thing").

The next turn was mine. (It was my turn.)

Text negative transfer. In Chinese writing, the text structure is relatively loose and casual, so there is not necessarily a topic sentence for each paragraph. But in English, the structure of a paragraph must be clear with a topic sentence. So a lot of Chinese students would write this kind of paragraphs in English, such as paragraph without clear topic sentence, or without logical connection between two paragraphs. Within one paragraph, cause-and-effect connection words, chronological words would be absent.

Cultural negative transfer. Chinese and English have greatly different cultural background, and this will lead to the result that students living in Chinese cultural background would show this in their English writing, for example, the position where they put "I" in subject when there are more than one person in subject. And they always try to get a moral conclusion in the writing.

\section{The enlightenment of the study in college English teaching}

\section{To guide the students properly to know about negative transfer}

Teachers should know more about negative transfer and then guide the students properly to understand the errors caused by mother language in writing. Teacher are expected not just to correct the students' mistakes, but to instruct them to know the origin and developing of negative transfer, so that students could treat it in a correct way or even to avoid them in their writing.

\section{Enhance the students' language input}

In college English teaching, teachers should try to guide students to enlarge their vocabulary, and let them grasp the correct usage of the words, as well as collocation. Let them know the correct sentence structure and text organization. Meanwhile, encourage students to memorize more passages written by native speakers, classify the students' errors while correcting them, and let the students know the errors caused by negative transfer of mother language. Their own awareness of the errors would help more in 
their learning, so teachers can encourage students to correct each other's writing and try to find out this kind of errors.

\section{Enhance the students' cultural input}

For students who live in Chinese culture, intentional cultural input is quite necessary. English cultural input in class is an effective way, and students should be encouraged to supplement their cultural knowledge after class. Interesting class activities are also a way to strengthen students' cultural knowledge.

\section{Summary}

From what we have discussed above, negative transfer from mother language is common and unavoidable. It affects college students' English writing in more than one way. So teachers are expected to guide students to know about negative transfer from mother language, as well as to encourage them to enhance their own language skills in vocabulary, sentence structure, and text organization. And thinking in a native English way is more important. Intentional cultural input also plays a significant role in it.

\section{Acknowledgements}

This research is assisted by Xidian University; 7214491701

\section{References}

[1] Corder S. P. Error Analysis and Interlanguage [M].

[2] Ge LiLian, The Impact of "Mother Language Transfer" on Chinese Students' English Expression, Journal of Capital Normal University ( Social Sciences Edition), P50-52 [J]

[3 ] Corder S. P. Error Analysis and Interlanguage [M].

[4] Zhang LinLin, A Study on Negative Cultural Transfer of Mother Language in College English Writing, Journal of Nanyang Institute of Technology, P51-55 [J] 2012 (4)

[5] Tang Ting, The Influence of Difference in Thinking Between East and West on Second P76-78 [J] 2013 (6), Journal of Southwest Agricultural University ( Social Sciences Edition) . 